\title{
Josephson Fourier Spectrometer Based on HTSP: Construction and Quantum Computer Realization Problem
}

\section{Dresvyannikov Maxim, Zherikhina Larisa, Murzin Vladimir, Tskhovrebov Andrey}

The Lebedev Physical Institute of the Russian Academy of Sciences, Moscow, Russia

Email: dresvjannikovm@gmail.com

Received 17 June 2014; revised 19 July 2014; accepted 5 August 2014

Copyright (C) 2014 by authors and Scientific Research Publishing Inc.

This work is licensed under the Creative Commons Attribution International License (CC BY).

http://creativecommons.org/licenses/by/4.0/

\section{(c) (i) Open Access}

\section{Abstract}

The possibility of creating a spectrometer of THz range, which is based on non-stationary Josephson effect in HTSP is considered. Simple design of Josephson junction adjustable at cryogenic conditions is proposed. Using of such device for solving the problem of factorization of high value numbers, which in fact is the only motivation for creating a quantum computer, is discussed.

\section{Keywords}

High-Temperature Superconductors, Josephson Effect, Quantum Computer

\section{Introduction: Non-Stationary Josephson Effect in HTSC Systems and Its Applications}

Previously we have investigated non-stationary Josephson effect via dot junction, which was performed by crossing of edges of two prisms of $\mathrm{Y}_{1} \mathrm{Ba}_{2} \mathrm{Cu}_{3} \mathrm{O}_{7-\delta}$. Josephson effect [1] is a phenomenon, where it is possible to observe persistent and phase-dependent current in tunnel structures (weak link):

$$
\begin{gathered}
I_{J}=I_{c} \sin \varphi \\
I_{c}=\frac{\Delta}{2 \pi \mathrm{e} R_{T}} \operatorname{th}\left(\frac{\Delta}{2 k T}\right) \\
\frac{\partial \phi}{\partial t}=\left(\frac{2 \mathrm{e}}{\hbar}\right) V
\end{gathered}
$$

where $I_{c}$-critical current, $\varphi$ - “phase difference” across the junction, $V$-voltage, $R_{T}$ - tunneling con- 
ductance. In our case junction is exposed to radiation, thus we have: $V(t)=V_{0}+v \cos (\omega t)$ which gives us the next equation for Josephson current:

$$
I_{j}=I_{c} \sum_{n=-\infty}^{n=+\infty} J_{n}\left(\frac{2 \mathrm{e} v}{\hbar \omega}\right) \sin \left\{\left(n \omega+\frac{2 \mathrm{e}}{\hbar} V_{0}\right) t+\phi_{0}\right\} .
$$

From this equation, it follows that under irradiation of Josephson junction it is possible to observe steps on a current-voltage characteristic with $j_{c} J_{n}\left(\frac{n v}{V_{0}}\right)$ amplitude. The experiment conditions corresponded to so-called inverse non-stationary Josephson effect with external electromagnetic microwave radiation (in our case $f_{e x t} \approx$ $140 \mathrm{THz}$ ). We observed Shapiro steps on the curve of the current-voltage characteristic that pointed on an appearance of Josephson effect [1] [2], and enabled drawing a conclusion about high microwave radiation sensitivity of the contact (Figure 1). Also it's worth mentioning that our junction is cheap and easy to produce; that's very important in case of breakdown. So it is viable to construct a fast spectrum analyzer with the high microwave radiation sensitivity based on HTSP Josephson junction [3]. Such a device could record THz radiation, as $2 \pi \hbar f_{e x t}<\Delta$, and $\mathrm{Y}_{1} \mathrm{Ba}_{2} \mathrm{Cu}_{3} \mathrm{O}_{7-\delta}$ possesses a wider superconductivity gap $\Delta$ in comparison with traditional superconductors. The action of the analyzer comes to recording current-voltage characteristic, recognition of Shapiro steps, and restoration of signal spectrum based on them.

\section{Method of Analogy Simulating of the Quantum Computer with the Help of the Single Josephson Contact: Discussion of the Scheme of the Device}

One of the most interesting modern problems, which demand quick production of Fourier transform, is the realization of quantum computer [4]. At present, there are a lot of research centers, which try to find ways of realization of quantum computer. Yet, there is the only applied problem for such a devise. It is the problem of factorization of multidigit numbers, which provides the framework for code-breaking of the high defensive codes in cryptography [5]. The method of factorization is based on the classical Euclid algorithm [6]. For factorization of an integer number $N$ on prime factors it is necessary to find periods of the function $f(x)=a^{x} \bmod N$, where $A$ mod $B$ is the residue of division $A$ by $B, x$-natural number. Then one takes the lowest period $r$, and defines the proper divisor $N-\operatorname{gcd}\left(a^{\frac{r}{2}+1}, N\right)$, where $\operatorname{gcd}(A, B)$ is the greatest common divisor of $A$ and $B$.

This simple algorithm for solving the problem of factorization of numbers could be realized with the help of quantum computer. As the period of function $f(x)$ corresponds to the maximum of its Fourier-spectrum, it is enough to find appropriate coordinates of the extremum. The quantum computer permits to do such operation for a few measurements of $X$ register. Registers $X$ and $Y$ form entangled state, when all combinations of $q$-bites, which correspond to domain of $x$ definition, are present at the $X$, due to quantum fluctuation and appropriate values of $y=f(x)$ are in register $Y$. We propose alternative approach for finding an effective substitution of the non-polynominal algorithm, which is based on the use of a spectrum analyzer with Josephson HTSP junction. In our case the role of quantum parallelism is substituted by the modeling of function $f_{N}(x)=a^{x} \bmod (N) \Rightarrow a^{t / \tau} \bmod (N)=f_{N}(t)$. Indeed, as a periodic function $f_{N}(t)$ contains the whole superposition (not quantum) of Fourier harmonics, which form this function supposing time $t$ is fixed, when it is multiple of the period $\tau$. In some way this is an analog of the quantum parallelism, when register $X$ of a quantum computer contains in the form of superposition all possible values of $x$ due to indefinite states of all $q$-bits. The block scheme of such device, which includes Josephson junction and is intended for factorization of numbers on prime factors, is presented on Figure 2. Here, voltage $U$ is applied to M1, thus the output of integrator is $U(t)=U * t$. Further, in accordance with Richardson formula we observe current $I(t)=I_{0}\left(\mathrm{e}^{\mathrm{e} U t / k T}-1\right)$ on diode $D$, and by zero shifting of integrator M2 we can compensate term $I_{0}$ in order to receive $V(t)=I_{0} R \mathrm{e}^{\mathrm{e} U t / k T}=a^{t}$ on M3 input (we assume for convenient that $I_{0} R=1$, thus $a=\mathrm{e}^{\mathrm{e} U / k T}$ ). Next we compare $V(t)$ with the analog signal $N$ (it is generated via M7) on comparator M4. If $V(t)$ exceeds $N$, then M4 generates pulse with amplitude, which is defined by M7 and M5. These pulses form step stair function after integrating on M6. Difference between signal $V(t)=a^{t}$ and step stair function is residue- $a^{t} \bmod (N)$, 


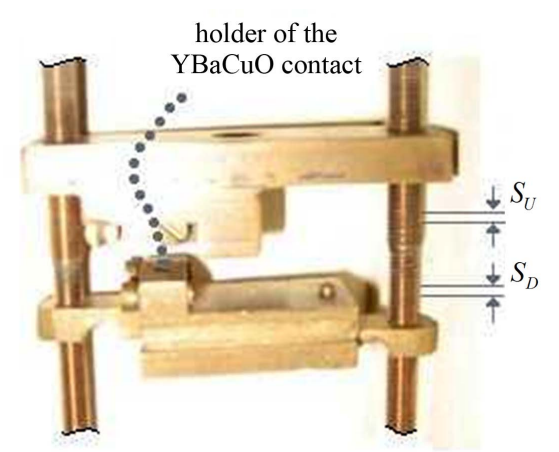

Figure 1. Right- and left-hand bolts are identical constructively. They have different thread pitch between higher and lower parts, that allows to displace electrodes of HTSP toward/outward by $S_{u}-S_{d}=50 \mathrm{~m} \mathrm{~cm}$ per one turn. Junction is shifted out of center for additional accuracy of adjustment of contact, that permit to use principle of lever.

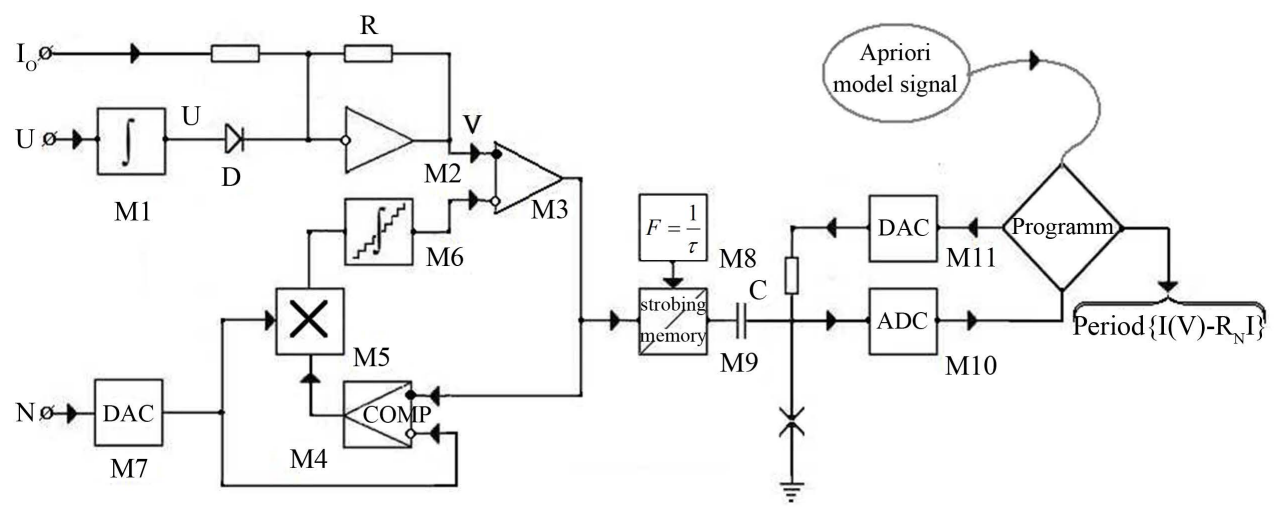

Figure 2. The block scheme demonstrates the realization of process of factorization on prime factors via Fourier spectrum analyzer, which is based on HTSP Josephson junction. Left-hand out of the capacity $C$ (M1 - M8)-block of formation of $f_{N}(t)=a^{t / \tau} \bmod (N)$. Right-hand out of $C$ (M9 - M11) - Josephson spectrum analyzer. M1-integrator with a constant voltage at the entrance; M2-integrator of currents; M3 - wide band differential operational amplifier; M4 - comparator; M5 — digital analog multiplier; M6-stepped integrator; M7-digital-to-analog converter; M8-tact generator; M9—strobe storage memory; cross-Josephson junction; M10 — digital-to-analog converter; M11—analog-to-digital converter.

which is needed for prime numbers factorization. From M3 residue is applied to M8, and after discretization it is applied to Josephson junction, where Fourier conversion is occurred.

\section{Conclusion}

Of course, during development, analog computing systems yielded to digital one in produced calculations. However, in our opinion in the field of quantum computers that moment will not come very soon.

\section{Acknowledgements}

This work was supported by the program "Strongly Correlated Electrons in Semiconductors, Metals, Superconductors and Magnetic Materials" of the Department of Physical Sciences of the Russian Academy of Sciences (project No. II-3).

\section{References}

[1] Josephson, B.D. (1962) Possible New Effects in Superconductive Tunnelling. Physics Letters, 1, 251. http://dx.doi.org/10.1016/0031-9163(62)91369-0 
[2] Shapiro, S., Janus, A.R. and Holly, S. (1964) Effect of Microwaves on Josephson Currents in Superconducting Tunneling. Reviews of Modern Physics, 36, 223. http://dx.doi.org/10.1103/RevModPhys.36.223

[3] Larkin, S. (1999) Measuring the Frequency of Monochromatic Microwave Field on the Basis of the Non-Stationary Josephson Effect. Research and Production Concern “Nauka”, National Research Center "Phonon”, Naukova Dumka, 271.

[4] Feinman, R.P. (1986) Quantum Mechanical Computer. Foundations of Physics, 16, 507. http://dx.doi.org/10.1007/BF01886518

[5] Vasilenko, O.N. (2003) Theoretic Algorithms in Cryptography. Moscow Center for Continuous Mathematical Education, Moscow.

[6] Berezkina, E.I. (1963) Mathematical Treatise by Sun Tzu. Historical and Mathematical Research, 3. 
Scientific Research Publishing (SCIRP) is one of the largest Open Access journal publishers. It is currently publishing more than 200 open access, online, peer-reviewed journals covering a wide range of academic disciplines. SCIRP serves the worldwide academic communities and contributes to the progress and application of science with its publication.

Other selected journals from SCIRP are listed as below. Submit your manuscript to us via either submit@scirp.org or Online Submission Portal.
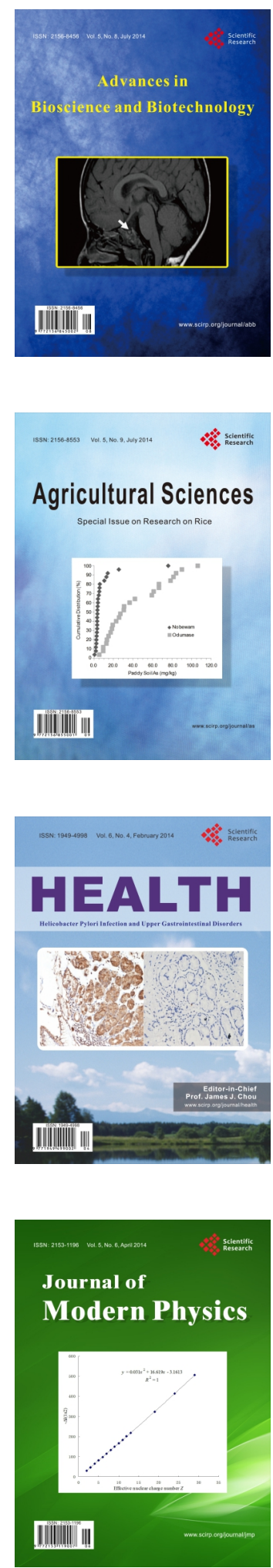
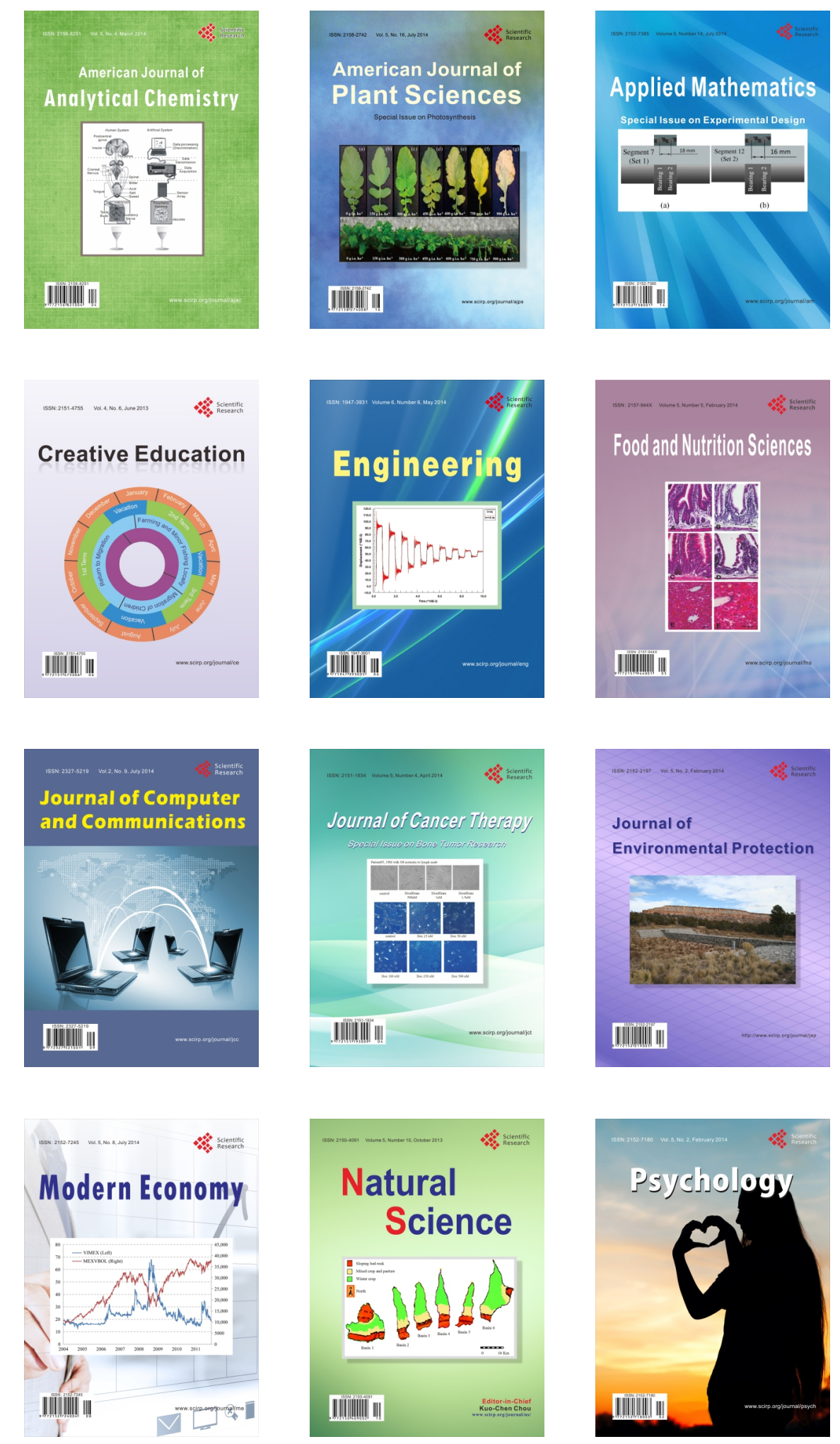